\title{
A Case study on Enterprise Collaboration and Interoperability in the Cloud Simon Oman
}

IT department of Polycom Škofja Loka, Poljane 76, Poljane nad Škofjo Loko 4223, Slovenia

$$
\text { simon.oman@polycom.si }
$$

\begin{abstract}
Increasingly, current software enables large and small companies to interact via cloud computing. Services offered in the cloud facilitate collaboration between databases and are a part of the information infrastructure that allows companies and organisations to move or integrate data within the cloud. This paper presents an analysis of the use of open source services that support cloud-based enterprise collaboration and interoperability. A practical case of an application of enterprise collaboration in the field of product development and production planning in the Slovenian automotive sector is presented. The results of solutions enable small and medium-sized companies, which have limited financial assets, to purchase one of the legacy systems and to become integrated within the supply chain of the automotive cluster of Slovenia. At the same time, the paper strives to establish the difference, if any, between conventional collaboration among companies and enterprise collaboration and interoperability in the cloud.
\end{abstract}

\section{Indexing terms/Keywords}

Enterprise collaboration, open source services, cloud computing, interoperability, case study.

\section{Academic Discipline And Sub-Disciplines}

Computers: Software Risk; Information Technology: Outsourcing, Offshoring; Software Life Cycle: Migration

\section{SUBJECT CLASSIFICATION}

Software Management: Software development, Software process

\section{TYPE (METHOD/APPROACH)}

Survey; Case Study

\section{Council for Innovative Research}

Peer Review Research Publishing System

Journal: INTERNATIONAL JOURNAL OF COMPUTERS \& TECHNOLOGY

Vol 13, No. 1

editor@cirworld.com

www.cirworld.com, www.ijctonline.com 


\section{INTRODUCTION}

In the last decade, the economy has become transformed into an extremely competitive market, because increasingly advanced and innovative information technologies enable the transformation of supply chains (Panetto and Molina, 2008) into global production chains. A decentralised approach to production (Mourtzis, 2011) has developed with the support of internet, which helps to coordinate production networks more efficiently (Abele et al., 2006). Modern companies continuously endeavour to improve competitiveness in the market with efficient strategic operations between different business partners. If great emphasis was paid to the optimisation of production processes in the last decade, now has come a period of rapid and more efficient synchronisation between different business units in the cloud (Zhang et al., 2011).

Hereafter, the paper presents an industrially solution, which is based on open source Enterprise COllaboration and

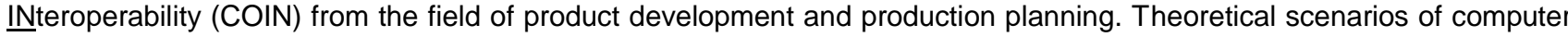
services in the cloud pose a question as how best open source solutions in the industrial sector should be introduced in the complex business environment. Advanced companies integrate information technology (IT) in their business processes, ensuring information flow from the production area to management. All the aforementioned software technology is connected with a comprehensive integration, which is the so-called Enterprise Application Integration.

\section{LITERATURE SURVEY}

A number of research studies have been conducted in the area of enterprise collaboration. However, there has been collaboration research addressing multi-objective nonlinear integer programming model and a genetic algorithm-based solution procedure for participated companies to maximize their profit (Ko, 2013). The results of corporate firm-level knowledge show that, compared to engineering manpower outsourcing (EMO), firm-level knowledge accumulation (KA) affords a greater degree of influence on the effectiveness of firm-level knowledge (Lai, 2011). Researchers (Jung et al., 2012) has been developed a methodology of collaborative performance measurement for manufacturing collaboration to evaluate the overall performance of a collaboration case which multiple manufacturing partners participate in.

Many researchers are fighting with the question of how efficient business cooperation is within the entire supply chain. They have been identified (Schall et al., 2008) different trends of cooperation, such as internet computer services in the cloud. Activities performed in the cloud are considered services, which can be divided into "Software as a Service", "Platform as a Service" and "Infrastructure as a Service" (Tao et al., 2011). In research (Vysotska and Chyrun, 2013) of commercial Web content processing subsystems are constructed a new approach of business processes application and implementation for the construction of electronic content commerce systems.

Hereafter, this research is related to the COIN Project (EU FP7 Project 216256), the basic objective of which has been to develop open source solutions that allow companies within the supply chain to cooperate. Such cooperation is usually supported by IT business platforms, which allow different sectors to communicate (COIN Consortium, 2010). The scenario presented in this paper, is related to data migration (technical and commercial data) in the cloud, where virtual cooperation or a so-called Virtual Organisation is established. In such a way, a group of developers is able to exchange opinions and recommendations on a 3D model, while commercial services exchange planned orders. The problem arising from the aforementioned, relates to the integration of different software (Oman, 2011) and primarily poses a question as how best the cooperation among companies, which use different software for 3D modelling and planning, may be improved (Weichhartet al., 2009). Moreover, particular attention needs to be paid to small and medium-sized companies wishing to participate in the development projects of larger companies and for which the purchase of software entails substantial costs. The purchase and maintenance of software represents a considerable financial burden; however, the aforementioned software requires highly trained staff. Therefore, the process of standardisation of business processes is required if companies wish to cooperate (Sitek et al., 2010).

The methodology relates to the knowledge and work method related to the implementation of the COIN Project, which is based on two main aspects: (AS-IS) and (TO-BE). On the one hand, the existing (AS-IS) model demonstrates the current state of business processes, which represent the basis for their transformation. On the other hand, the examined theoretical grounds (tools, analysis methods, modelling of business processes and their automation) represent a starting point for the improvement of the transformed processes (TO-BE). Based on additional features, boosted by integrated tools for process modelling, their advantages and use in industry shall be analysed. At the same time, the theoretical basis oriented towards the importance and role of open source solutions in industry is presented. The research is based on the process of introducing open source solutions into the industry and this implementation is performed through the application of open source services developed in the COIN Project, within an appropriately modelled workflow. This is followed by a discussion and recommendations for further work.

\section{CASE STUDY OF COIN SERVICES IN THE CLOUD}

\section{Theoretical framework of COIN services}

Open Source solutions were developed for the purposes of providing an alternative pragmatic ideology or FreeSoftware, which is rapidly turning into a business opportunity (Fitzgerald, 2006; Capra et al., 2011). Companies cooperating in open source projects usually contribute specific software. Porenta and Ciglarič (2013) believe there should be some additional fine-tuning of open-source algorithms. This simple model of cooperation enables companies to influence individual activities in project implementation. Contributing software allows companies to obtain a particular status in the projects and provides successful interactions with other project members (Ghosh et al., 2008). Based on different research studies addressing open source solutions, it is found that the introduction of their services in companies has increased in recent years (Ven and Mannaert, 2008). The solution demonstrated here, represents a new mental dimension, because open source services are used for product planning and development (e.g., orders, recalls, 3D models). Companies are integrated into business 
processes with an appropriate incorporation of the aforementioned service (Sesana et al., 2010). The users are thereby divided into two groups:

- Legacy system users.

- Non-legacy system users.

Legacy system users are those who use one of the legacy systems (e.g., SAP, Navision, Baan, Pro/Engineer, Catia), whereas non-legacy system users have none of the aforementioned systems available to them, only Internet Explorer. These are primarily users from small and medium-sized companies that wish to integrate into the supply chain to ensure continuous growth and development. As mentioned before, the users may be divided into legacy system and non-legacy system users; therefore, the method of integration between individual partners is different. The characteristic of the legacy system users (Figure 1) is that both parties (supplier and customer) prepare the data via File Transfer Protocol (FTP) in advance.

\section{C3P = Collaborative Production Planning Portal}

Agreed

C3DDS = Collaborative 3D Design Development

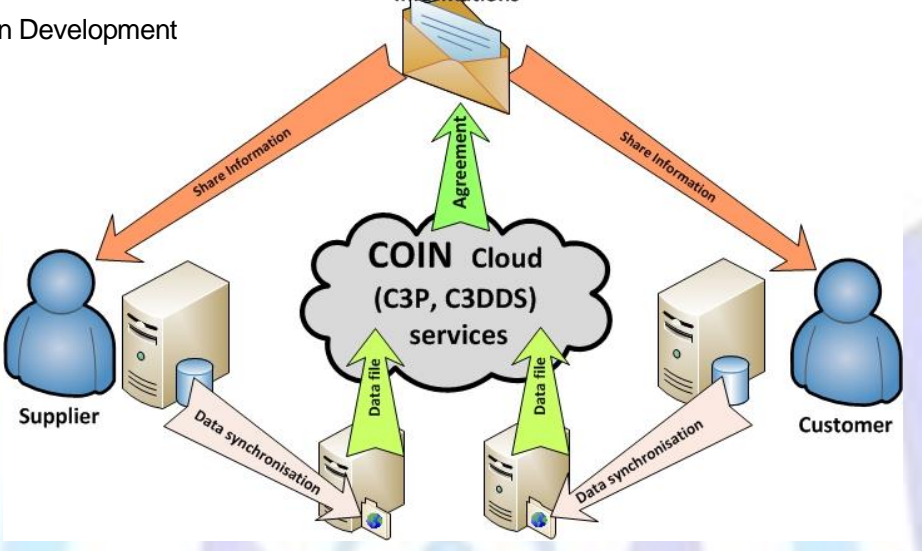

Figure 1: Exchange of information appertaining to legacy system users

Such data are than imported into one of the open source solutions. This is followed by the synchronisation of information in the so-called virtual organisation located in the cloud. All involved in a particular process enter into the virtual organisation and exchange opinions. When the opinions are synchronised, information on the agreement is distributed via electronic mail. Synchronisation between non-legacy system users (Figure 2) or users using only an internet browser is performed in a somewhat different manner.

\section{$\mathrm{C} 3 \mathrm{P}=$ Collaborative Production Planning Portal}

C3DDS = Collaborative 3D Design Development
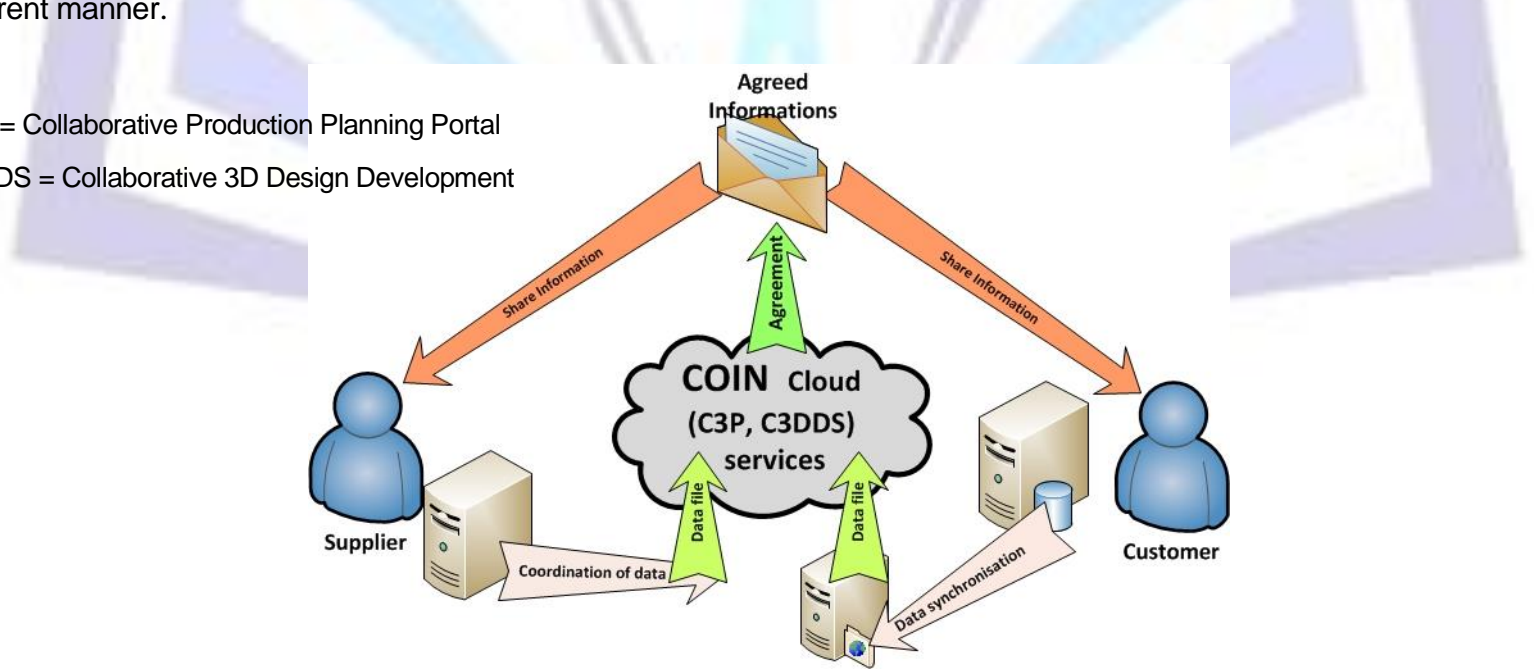

Figure 2: Exchange of information between legacy and non-legacy system

Legacy system users and non-legacy system users act in the process on the buyer's side, i.e., they prepare data on the FTP server, which are then imported into one of the open source solutions. In doing so, non-legacy system users access the virtual organisation via the internet browser, which is also located in the cloud. The procedure of synchronisation and exchange of opinions is the same as in the case of legacy system users; however, it is not necessary to use one of the legacy systems. With such an approach, small and medium-sized companies may integrate into the supply chain or virtual organisation. 


\section{Implementation of COIN services in the cloud}

For the purposes of the COIN project, the Slovenian Automotive Cluster (ACS) designed a live laboratory in which researchers, developers and users connect. Individuals in the live laboratory are experts from the ACS, and they form the demonstrator team. In addition to the aforementioned knowledge, the individuals also have knowledge on methodological approaches and organisational aspects. Cooperation in such labs may be seen by the individuals and companies as an opportunity for improving innovative integrated solutions from the field of open source systems, which are to be introduced gradually and are available for use in the real environment. Partners participating in the demonstrator represent different profiles, e.g., technical staff working in the fields of mechanical engineering, informatics and economics. The demonstrator team's researchers from the field of mechanical engineering boast competence and skill in the field of 3D modelling. With their knowledge, the mechanical engineers strive to verify whether the 3D model, exchanged with the help of the Collaborative 3D Design Development Services (C3DDS), is of sufficient quality to be used for further work (e.g., simulation, production of injection tool construction). The economists (planning and controlling departments) endeavour to introduce the Collaborative Production Planning Portal (C3P) services related to planning. Their task is to ensure a uniform planning table, which enables planning activity for all involved in the supply chain. The task of the IT staff is to provide an interface to enable data exchange in a user-friendly manner. The demonstrator team agrees that data exchange is performed with the help of swap files. The joint task of the demonstrator team is related to the preparation of workflows with which the process of cooperation is unified. All demonstrators strive towards a joint objective; to introduce data exchange to the processes of cooperation using the simplest method possible. The demonstrators in the supply chain test two scenarios: a "Receive order from customer" scenario (Figure 3) and a "Release demand for components" (Figure 4). The design of workflows is performed using an open source tool for modelling the process called ProcessMaker. The results related to the modelling of workflows focus on data exchange and they are demonstrated in the following.

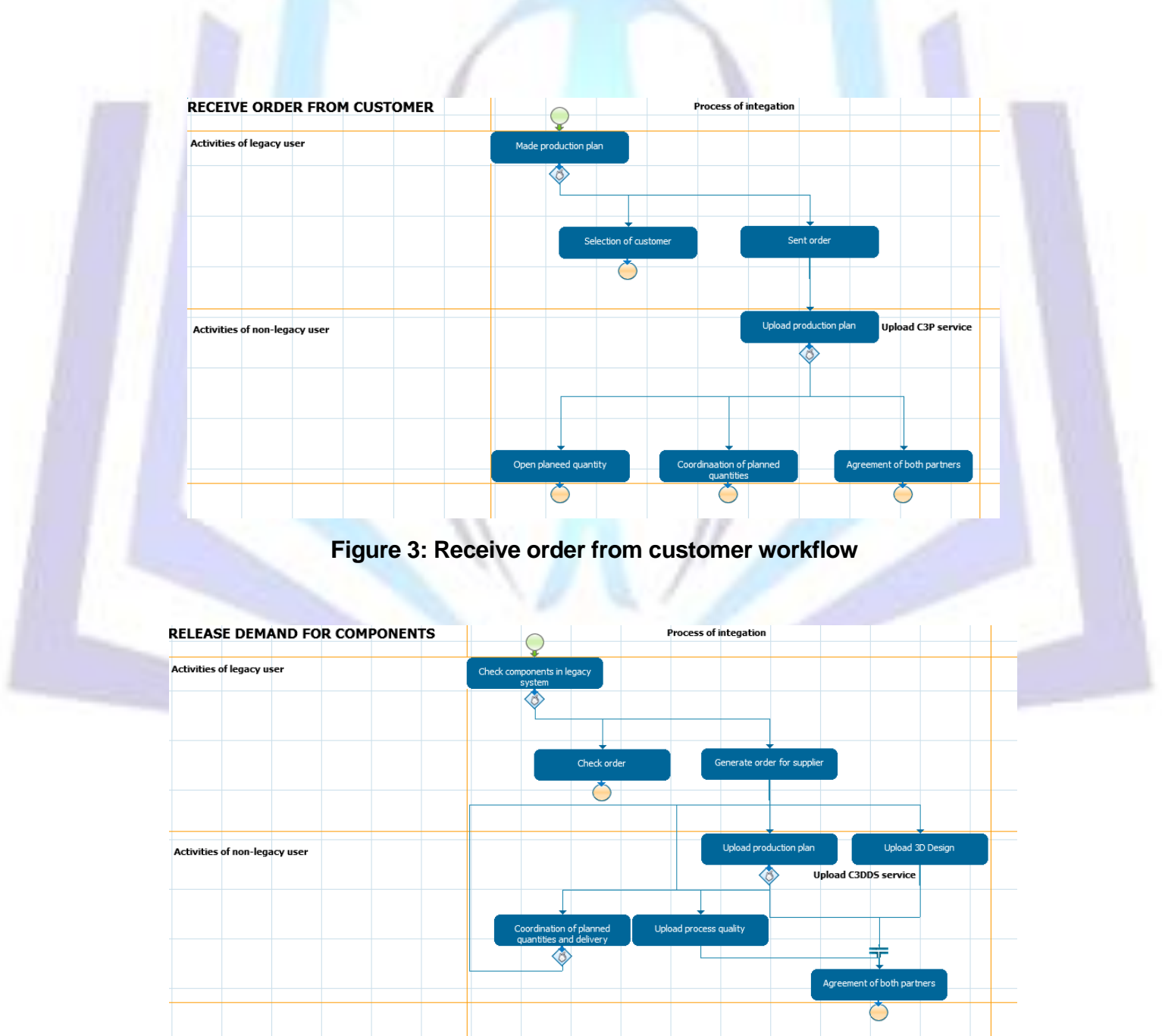

Figure 4: Release demand for components workflow

In the existing scenario (AS-IS), the "Receive order from customer" workflow is an Excel table, including products of individual suppliers, which is prepared once a day. This table is then sent to the suppliers via electronic mail. By developing the workflow, a modelled process is prepared within ERP. The developed interface is triggered automatically in each work shift and it provides data exchange between buyer and supplier. Although data may be transferred automatically during 
each shift, the capability for manual transfer of orders from buyer to supplier is also provided. For this purpose, the trigger for the transfer of an order from the buyer on the FTP server is activated manually. In such a way, data are prepared in advance for joint use and intended for import into the C3P service. Contrary to the "Receive order from customer" scenario, which is oriented towards the exchange of commercial data, the "Release demand for components" scenario is more technically oriented. This scenario represents the demand for the development of a product, whereas a 3D model is used in the majority of cases, representing a constant for companies in the automotive sector. The scenario focuses on the exchange of 3D models between legacy system users and non-legacy system users. It should be mentioned that the export of the 3D model is performed using the "Save as" function in one of the legacy systems (e.g., Pro/E, Catia). Furthermore, in this case, the file is saved on the FTP server and is intended for export into the C3DDS. The solutions demonstrated hereafter represent the use of open source services in the industry. The first example demonstrates the cooperation in the cloud, where synchronisation of announced quantities and agreed prices is performed in the virtual organisation. In the second example, the cooperation in the cloud relates to the agreement of the 3D model, where synchronisation is oriented towards product development.

\section{Product planning in the cloud}

Production planning in the cloud represents the transfer of a certain quantity of data from the legacy system (in most cases the business information system, ERP) to a particular location in the cloud. This entails the transfer of not only those data that the open source needs for the planning horizon (usually this is a required quantity and date, as well as a price, unless the price has already been agreed with a contract). In order for the transfer to take place, appropriate data need to be exported to a required location beforehand; in our case, the FTP server. Data may be recorded in differed formats (e.g., txt, csv, xml.) depending on the future use of the file. This file, which is created from the legacy system, actually represents the Make-To-Order (MTO) for a supplier and is used for production planning. In the next step, the data are exported to the open source service, accessible by all interconnected partners who cooperate in the virtual organisation (TO-BE). In such a way, partners create a joint planning table, which allows all those involved in the production process to monitor the demand for products and deadlines. At the same time, the table discloses by what time and with which quantity the products need to be finished. In such a way, the agreement and synchronisation process on one spot is made possible (Figure 5). When individual partners reach an agreement between themselves, a confirmation on the agreement is issued, which is usually received via electronic mail

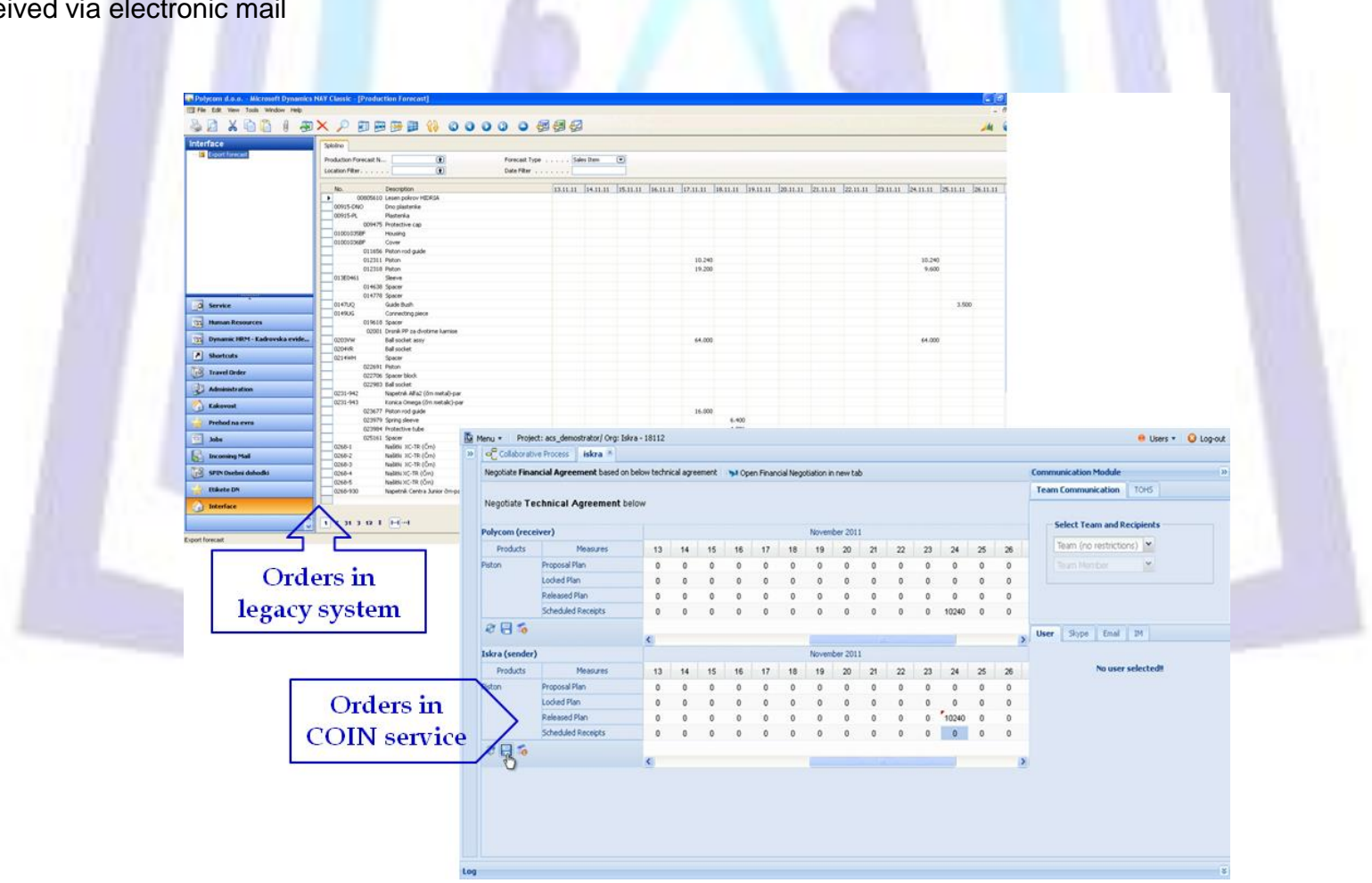

Figure 5: Comparison of planning in legacy system in the C3P open source service

\section{Product development in the cloud}

Product development in the cloud represents an upgrade of the existing model, which is predesigned in a legacy system. Following the usual work method (AS-IS), the model transfer between the users is performed by converting the 3D model into the STEP (STandardised Exchange of Product) or IGES (Initial Graphics Exchange Specification), as well as the VDA (Verband der Automobilindustrie) format. Thus, a file is created that is then sent via electronic mail. However, a problem arises with the aforementioned formats (STEP, IGES, VDA), i.e., different models interpret import data differently. This is 
particularly evident with the 3D models exchanged between different legacy systems. From a technical point of view, there is a certain gap in the import of data from the aforementioned formats between particular surfaces (Ming et al., 2008), which in further use might cause a somewhat distorted surface on the originally designed 3D model. Therefore, a solution is required to be found, i.e., a solution that will enable data exchange in a standardised manner (TO-BE). Two formats are available: STEP and STL (Standard Tessellation Language). Technology is developing rapidly in all fields; however, the STL format is defined in research as the standardised record for 3D model exchange. Thus, the STL format is selected owing to its multi-functional application. The first purpose is most definitely to ensure a unified format or a standardised method of 3D model exchange. A graphical demonstration of 3D model exchange is presented in Figure 6 . The second purpose of using the original file in the STL format is the Moldflow simulation, which is used in tool construction and parameter setting of a machine for injection moulding. The third purpose of using the original file in the STL format is the printing of 3D models. The technique of printing 3D physical models is intended for all those who need the models for visual presentations, ergonomic studies and useful prototypes, although they may also be used for demonstrating the adequacy of particular sets.

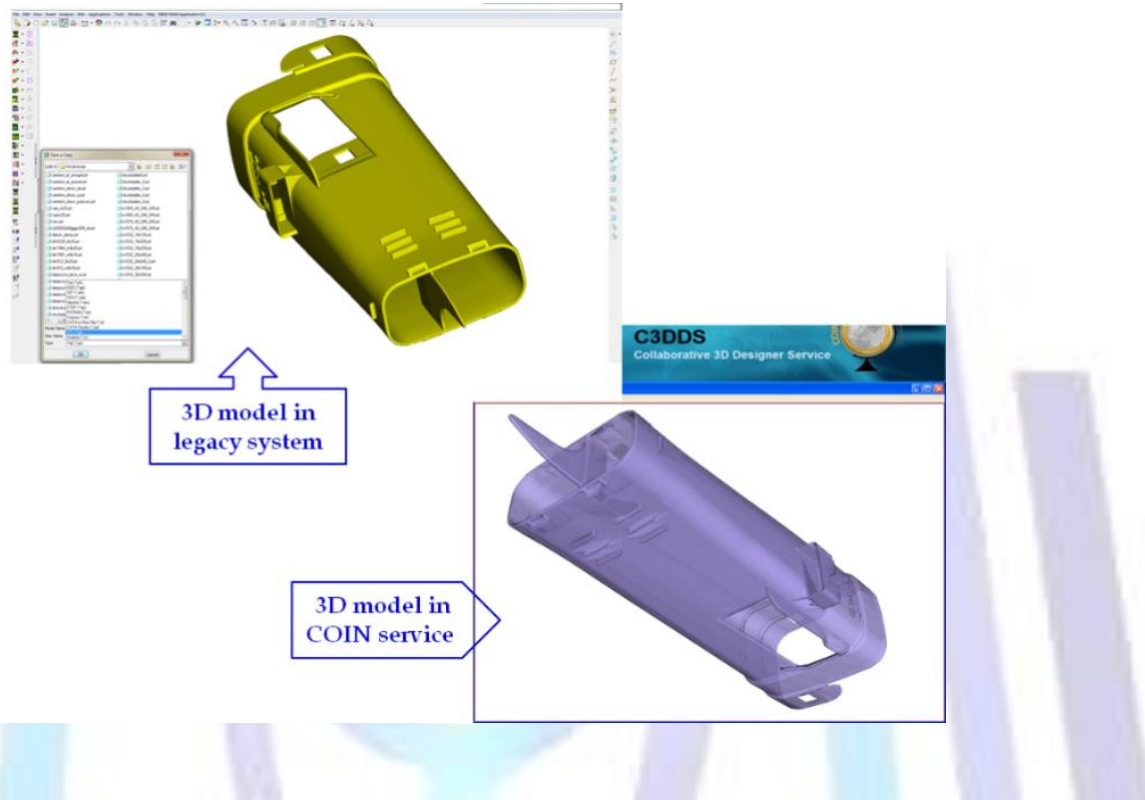

Figure 6: Comparison of product development in legacy system in the C3DDS open source service

\section{DISCUSSION}

Computing in the cloud represents the combining of two principal trends in IT (Marston et al., 2011):

- Efficiency; the power of modern computers and their extendable services is used.

- Business agility; IT may be used as a competitive tool with swift installation, parallel processing, useful business analysis and mobile interactive applications, which respond to users' needs in real time.

Computing in the cloud has been recognised worldwide as a new and promising platform for providing information infrastructure and services. Users may access these services based on their personal and business needs; however, questions regarding the protection of privacy are often expressed (Jun and Wei, 2011), because there is no control over what is happening inside the cloud. Consequently, the protection of privacy is becoming one of the most important issues for further research (Chonka et al., 2011). Irrespective of the problems of cooperation and interoperability (privacy protection) entailed by computing in the cloud, the solution, with the services presented in the paper represents an important step in the development of cooperation in the entire automobile industry, not only in Slovenia but also internationally (Del Grosso et al., 2010). The services developed under the framework of the COIN project enable final users, which may be large as well as small companies, to interact in different cooperative processes. Cooperation facilitates a unified process (Stol et al., 2011), which is based on open source technology. Thus, a large number of companies may be attracted to cooperate, i.e., companies that do not use one of the legacy systems but use only an internet browser.

The innovative COIN services represent cooperation in managing business processes and workflows (Del Grosso et al., 2010). Therefore, companies are able to standardise their business processes more easily, which facilitates cooperation. The primary advantage of the COIN services is that by using appropriate software, business process management throughout the supply chain is ensured. Owing to a substantial increase in the importance of different information systems, 
the use of the COIN services in the economy is of great importance, because it provides simple use and access to these services from different locations. The COIN services entail the following advantages:

- Improvement in interoperability between partners of heterogeneous legacy systems.

- $\quad$ Provision of e-business between micro companies failing to boast legacy systems.

- Support provided to joint documents and files via joint cooperation platforms.

- Decrease in time and costs in the phase of joint product development.

- $\quad$ Support in cooperation among partners and provision of new business opportunities.

The testing of the COIN services in the live lab provides valuable knowledge related to the different fields of cooperation. At the same time, individuals in the demonstrator team orient towards the development of integration between different software tools and the management of workflows. Developed services reduce the investment by companies in different software tools, because the COIN services facilitate cooperation based on Internet technologies. Thus, it may be concluded that state-of-the-art IT will be based on supporting cooperation, by relying on open source solutions in the cloud. The aforementioned facts and demonstrated study represent an answer to the posed question regarding the difference between conventional cooperation between companies and their cooperation in the cloud.

\section{CONCLUSIONS}

The influence of the COIN innovative IT in the economy leads to the development of new technologically advanced industries. New technologies usually develop and expand rapidly because human activity is increasingly focused on the development of IT in manufacturing industries (Lai and Chen, 2009). Owing to its complexity, the developers of the COIN services invited users from industry to participate (Withalm and Wolfel, 2008) in developing services with appropriate synergy and to stimulate the development of small and medium-sized companies. The development of the COIN services includes modern information solutions that encompass different maintenance, software solutions of information systems, databases, scientific research work and suchlike. The COIN IT changes the product life cycle and its exchange between customers. Owing to the nature of its use, products change rapidly, which is felt most by producers of automobile components, because new models of vehicles are frequently introduced to the market. In doing so, the COIN IT with its innovative solutions may contribute substantially to the shortening of the product development cycle, which is usually measured from an idea to its placement in the market.

The ACS orients its plans towards the expansion of knowledge from the field of the COIN services and their introduction into the industry. In doing so, the ACS strives to increase the role of small and medium-sized companies in their integration into the supply chain. Above all, it strives to raise awareness on the importance of IT in manufacturing companies, which will contribute to the development of small and medium-sized companies, ensuring a successful and competitive environment in the global market.

\section{ACKNOWLEDGMENTS}

The author thank the partners and the European Commission for support in the context of the COIN Project under contract number EU FP7-216256. For more information, see http://www.coin-ip.eu/.

\section{REFERENCES}

[1]. Abele, E., Elzenheimer, J., Liebeck, T., Meyer, T. (2006). Reconfigurable Manufacturing Systems and Transformable Factories - Globalization and Decentralization of Manufacturing, (1st.edition) Springer, pp. 4-5.

[2]. Capra, E., Francalanci, C., Merlo, F., Rossi-Lamastra, C. (2011). Firms' involvement in Open Source projects: A tradeoff between software structural quality and popularity, The Journal of Systems and Software, 84(1), 144-161.

[3]. Chonka, A., Xiang, Y., Zhou,W., Bonti, A. (2011). Cloud security defence to protect cloud computing against HTTP-DoS and XML-DoS attacks, Journal of Network and Computer Applications, 34(4), 1097-1107.

[4]. COIN Consortium, (2010). Maturity Model for SME collaboration. Deliverable D6.3.b.

[5]. Del Grosso, E., Gusmeroli, S., Olmo, A., Garcia, A., Busen, D., Trebec, T. (2010, 27-28 October).Are enterprise collaboration and enterprise interoperability enabling innovation scenarios in industry? The COIN IP perspective in Automotive. Paper presented at the conference of the eChallenges, Warsaw-Poland.

[6]. Fitzgerald, B. (2006). The transformation of Open Source software. MIS Quarterly, 30(2), 587-598.

[7]. Ghosh, R., Haaland, K., Hall, B.H., (2008). Whichfirmsparticipate in open source software development? A studyusing data fromDebian. In: Proc. DIME - DRUID, Copenhagen, Denmark., Retrived 11/2/2012, from

http://elsa.berkeley.edu/ bhhall/papers/GhoshHaalandHall08 Debian.pdf. 
[8]. Jun,C., Yan Wei, M. (2011, 18-19 June). The Research of Supply Chain Information Collaboration Based on Cloud Computing, 3rd International Conference on Environmental Science and Information Application Technology ESIAT 2011, Beijing-China, 10 (A), 875-880.

[9]. Jung, J.Y., Lee, J., Jung, J., Kim, S.K., Shin, D. (2012). A Methodology of Collaborative Performance Measurement for Manufacturing Collaboration. International Journal of Industrial Engineering: Theory, Application and Practice, 19(3).

[10].Ko, H. (2013). Introducing the concept of autonomous agents as a tool to assist the analysis of the collaboration between manufactured exporters and maritime shippers. International Journal of Industrial Engineering: Theory, Application and Practice, 20(1-2).

[11].Lai, J., Chen, W. (2009). Measuring e-business dependability: The employee perspective. The Journal of Systems and Software, 82(6), 1046-1055.

[12].Lai, W.H. (2011). Corporate Firm-Level Knowledge Accumulation and Engineering Manpower Outsourcing. International Journal of Industrial Engineering: Theory, Application and Practice, 18(4).

[13].Marston, S., Li, Z., Bandyopadhyay, S., Zhang, J., Ghalsasi, A. (2011). Cloud computing - The business perspective, Decision Support Systems, 51(1), 176-189.

[14].Ming, X.G., Yan, J.Q., Wang, X.H., Li, S.N., Lu, W.F., Peng, Q.J., Ma, Y.S. (2008). Collaborative process planning and manufacturing in product lifecycle management, Computers in Industry, 59(2-3), 154-166.

[15].Mourtzis, D. (2011). Internet based collaboration in the manufacturing supply chain, CIRP Journal of Manufacturing Science and Technology, 4(3), 296-304.

[16].Oman, S. (2011). Application of intermediate document message in the process of enterprise resource planning and manufacturing executing system integration, Problems of Management in the 21st century, 2(2), 141-156.

[17].Panetto, H., Arturo Molina, A. (2008). Enterprise integration and interoperability in manufacturing systems: Trends and issues, Computers in Industry, 59(7), 641-646.

[18].Porenta, J., Ciglarič, M. (2013). Comparing commercial IP reputation databases to open-source IP reputation algorithms, Computer System Science and Engineering, 28(1).

[19].Schall, D, Truong, D.L, Dustdar, S. (2008). "Unifying human and software services in web-scale collaborations," Internet Computing, IEEE, 12(3), 62-68.

[20].Sesana, M., Taglino F., Cannas V., Gusmeroli S. (2010, 27-28 October), Production Planning and Knowledge Interoperability Utility and Value-Added Services in Aerospace Domain. Paper presented at the conference of the eChallenges, Warsaw-Poland.

[21].Sitek, P., Seifert, M., Thoben, K.-D. (2010). Towards an inter-organisational perspective to manage quality in temporary enterprise networks, International Journal for Quality and Reliability Management, 27(2), 231-246.

[22].Stol, K.J., Babar, MA.,Avgeriou, P., Fitzgerald, B. (2011). A comparative study of challenges in integrating Open Source Software and Inner Source Software, Information and Software Technology. 53 (12), 1319-1336.

[23].Tao, J., Marte, H, Kramer, D., Karl, W. (2011, 1-3, June). An Intuitive Framework for Accessing Computing Clouds, Paper presented at the conference of ICCS 2011, Singapore-Japan, 4, 2049-2057.

[24].Vysotska, V., Chyrun, L. (2013). Web contente processing method for electronic business systems. International Journal of Computers \& Technology. 12 (2),3211-3220.

[25].Ven, K., Mannaert, H. (2008). Challenges and strategies in the use of Open Source Software by Independent Software Vendors, Information and Software Technology, 50(9-10), 991-1002.

[26].Weichhart, G., Feiner T., Stary C. (2009). Implementing organisational interoperability-The SUddEN approach, Computers in Industry, 61(2), 152-160.

[27].Withalm, J. \&Wolfel, W. (2008, 16 September).Improvement model for collaborative networked organizations. Paper presented at the conference of Enterprise Distributed Object Computing Conference Workshops, Vienna-Austria.

[28].Zhang, G., Shang, L., Li, W. (2011). Collaborative production planning of supply chain under price and demand uncertainty, European Journal of Operational Research, 215(3), 590-603. 


\section{Author' biography with Photo}

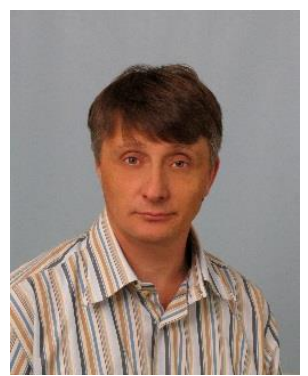

Simon Oman has been employed with the company Polycom d.o.o. since 2004. He is responsible for the field of informatics. He began his professional career in the field of mechanical engineering where he worked on different processes and techniques of cutting, CNC programming and tool construction for technical plastic materials. He continued with the postgraduate studies in the field of informatics and management where he focuses his research work on modernisation and computerisation of business processes which encompass the areas of ERP and MES systems. 